\title{
HOW CREDIBLE IS MY HAZARD MAP? DISSECTING A PREDICTION PATTERN OF LANDSLIDE SUSCEPTIBILITY
}

\author{
ANDREA G. FABBRI ${ }^{1}$ \& CHANG-JO CHUNG ${ }^{2}$ \\ ${ }^{1}$ DISAT, University of Milano-Bicocca, Italy \\ ${ }^{2}$ SpatialModels Inc., Canada
}

\begin{abstract}
A hazard map is to represent the likelihood of future occurrences of hazardous events. Predicting the future, however, is far from easy and prone to uncertainty, misunderstanding and suspicion. This contribution presents a landslide-susceptibility prediction pattern that resembles a map but is a complex construction resulting from modelling and assumptions imposed on historical data. A mathematical model of spatial relationships, based on fuzzy sets, is applied to a spatial database that was constructed for landslide susceptibility studies in northern Italy. The resulting prediction pattern is dissected into its basic components to bring up the implied elements of spatial characterization, relevance of thematic input, assumptions in models and data, and visualization/interpretation of predicted scores. By the term prediction pattern we indicate the result of the classification of the study area into levels of relative susceptibility. Of particular relevance within the study area is the uncertainty associated with the scores, i.e., the ranks that classify the zones that are relatively more susceptible. Such a dissection is to lead to a confirmation of the credibility of the prediction pattern (hazard map?) based on the confidence gained by the comprehension of all elements integrated and assumptions made in its construction.

Keywords: prediction pattern, landslide susceptibility, hazard map, uncertainty of ranks, modelling assumptions.
\end{abstract}

\section{INTRODUCTION}

With the availability of digital data, it has become feasible practice to construct spatial databases for hazard mapping and in particular for landslide hazard. In the databases the spatial distribution of known hazardous events is related with the distribution of categorical map units such as lithology, vegetation/land use or permeability classes, for instance. The event distribution is also related with that of continuous field values of slope angles, digital elevation and aspect angles, among others, in the hope of identifying situations that characterize the locations with presence of the hazardous events with respect to those with their absence within the database.

Mathematical models for representing indices of spatial relationship for each categorical or continuous field map are used to integrate their contribution in classifying the study area in the database into levels of likelihood of future occurrences. Many models have been proposed for establishing and integrating the spatial relationships and a variety of techniques are being used to map the likelihood obtained into convenient numbers of classes. While landslide susceptibility is the likelihood of a landslide occurring in an area in relation to local terrain characteristics, landslide hazard is defined as the probability of occurrence of a landslide of a given magnitude in a specific period, within a given area. Predicting the future, however, is far from easy and prone to uncertainty, misunderstanding and suspicion. Also, it has to be based on unavoidable assumptions. This contribution presents a prediction pattern of landslide-susceptibility that resembles a map but is a complex construction resulting from modelling and assumptions imposed on historical data on hazardous events. A mathematical model of spatial relationships based on fuzzy sets is applied to a spatial database that was constructed for landslide susceptibility studies in northern Italy. The resulting prediction 
pattern is dissected into its basic components of spatial modelling. The procedure discussed here is to represent an example of wide applicability.

The next section provides an instance of a landslide susceptibility map termed prediction pattern, i.e. the result of the classification of the study area into levels of relative likelihood of occurrence. The following section discusses the assumptions required in prediction modelling: on the databases, the spatial relationships modelled, and the mathematical model itself. Next is the interpretation and evaluation of the resulting prediction pattern, followed by a discussion on the credibility achieved by the pattern.

\section{INSTANCE OF LANDSLIDE SUSCEPTIBILITY PREDICTION PATTERN}

An example of prediction pattern of landslide susceptibility is introduced here in order to discuss its significance and credibility for decision making in safe land use planning. The prediction pattern in Fig. 1 appears as a map that shows classes of likelihood of landslide occurrence sequenced in decreasing order, as indicated by the legend, from hot to cold pseudo-colours. Each colour corresponds to groups of equal-area ranks of predicted landslide susceptibility scores. In black patches is the distribution of 70 active landslide occurrences, trigger areas, used in the modelling to obtain the likelihood classes. In the illustration their sizes are exaggerated for visibility. As can be observed, most but not all of the occurrences are located within the higher classes or hotter colours. We have used the term pattern because it consists of arbitrarily selected thresholds of continuous scores.

At this stage we do not yet know the predicting quality of the classification into relative ranks but, even before interpreting them, let us consider how the display of the ranks has been structured. Let us call $\boldsymbol{A}$ the study area that consists of 646,091 picture elements or pixels arranged in a rectangular array or raster, each pixel being assigned a numerical value corresponding to an aggregated score. The scores were sorted from maximum to minimum and 200 equal-area ranks were identified, each covering $0.5 \%$ of the pixels in $\boldsymbol{A}$. The ranges are visualized as pixel ranks, out of the 200 , that correspond to groups of $0.5 \%$ ranks used for the prediction pattern, each of about 3230 pixels. The higher ranks are displayed with narrower ranges $(1 \%, 2 \%, 2.5 \%, \ldots)$ being of greater interest than the lower ranks that are with wider ranges $(\ldots, 10 \%, 12.5 \%)$.

Let us now consider the content of the spatial database and the prediction model used for its generation. The pattern shown in Fig. 1 was obtained from the spatial database of the Tirano South study area in northern Italy constructed by Sangalli [1]. She provided an overview of its geomorphology and used the data for a study on spatial modelling of landslide susceptibility by applying a favourability model of empirical likelihood ratio function [2], [3]. For the function a unified mathematical framework for spatial prediction modelling has been developed by Chung and Fabbri [2]. The theoretical background for the empirical likelihood ratio function was conceptualized by Chung [3], who also introduced the procedural strategy of favourability modelling that is followed in this contribution. The same database studied in [1] was later described and used in experiments on comparing patterns from different prediction models [4]. The comparison demonstrated the greater predictive importance of database properties over those of the models, whose results showed only minor differences. One of the models was based on fuzzy sets as it is done here.

The location of the study area, $\boldsymbol{A}$, is shown in Fig. 2 along with the distribution of 70 landslide occurrences considered as active, i.e., directly observed as in motion or with seasonal occurrence characteristics. The pattern in Fig. 1 was obtained by a favourability model based on a fuzzy set membership function [5]. It used the distribution of the 70 active landslide trigger areas as a direct supporting pattern, DSP, to establish spatial relationships with units and values of several digital maps termed indirect supporting patterns, ISP. 


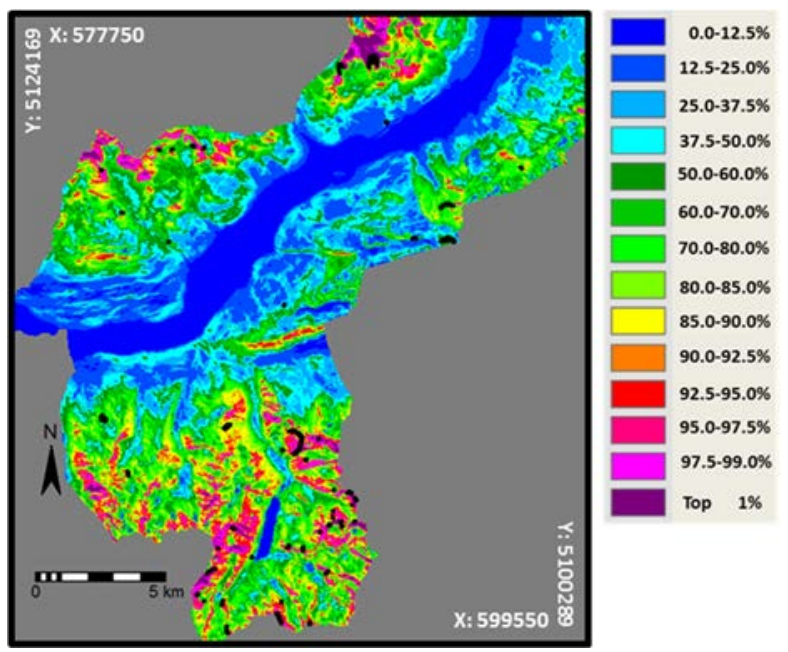

Figure 1: Fuzzy set function prediction pattern using seventy active landslide occurrences, 70at, as direct supporting patterns and ulpACDIS as indirect supporting patterns. Occurrences in black have exaggerated sizes for visibility. Pseudocolors in legend correspond to groups of equal area ranks. (Source: Modified after [4].)
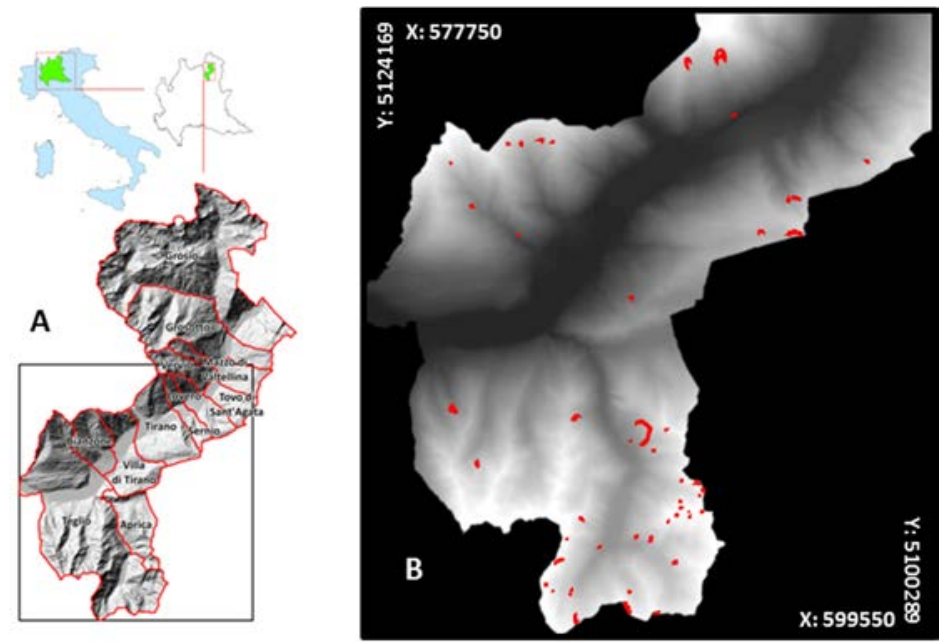

Figure 2: Location of the Tirano South study area in Lombardy Region, northern Italy in (A), and distribution of 70 active landslide occurrences in red in (B). Occurrences in red have exaggerated sizes for visibility. (Source: Modified after [4].)

The support is meant for a mathematical proposition linking DSP and ISPs of the type, " $\mathbf{T}_{p}$ : that a point $p$ in $\boldsymbol{A}$ will be part of trigger areas of future landslides | the presence of spatial evidence." 
Fig. 3 shows schematically the content of the data base as to the DSP and ISPs. The Tirano South database covers a rectangular area of pixels with $20 \mathrm{~m}$ resolution. It consists of 1090 pixels x 1194 lines and within it the $A$ occupies 646,091 pixels or approximately $258 \mathrm{~km}^{2}$. The availability of controlled geological and cartographical documentation including landslide inventories allowed constructing it with high quality of information [4]. The study area is of young Alpine geology with geomorphology controlled by glaciers with elevations from 200 to $4000 \mathrm{~m}$ a. s. 1., and strong climatic variations. Erosion due to torrent activity is predominant and it triggers landslides and mass movements in areas affected by deep gravitational deformation. Triggering cause is the convergence of several factors including intense rainfall and stream erosion at the valley bottoms where most anthropic activity takes place. Active and quiescent landslides were carefully mapped and described nevertheless it was not possible to separate rotational and translational landslides, so that this study deals with 70 active rotational-translational landslides in the $A$. They occupy 697 pixels (with average size of 9.81 pixels), representing $0.108 \%$ of the 646,091 pixels in the $\boldsymbol{A}$. Their distribution short named as 70at, shown in Fig. 2(B), has been used as DSP for the modelling.

The ISPs consist of three categorical digital maps of 25 land-use classes, 51 lithologic units and 8 permeability classes, short named as $\mathbf{u}, \mathbf{I}$ and $\mathbf{p}$, respectively. Classes and units are listed in Table 1. Other continuous field ISPs include aspect $\left(0^{\circ}\right.$ to $\left.359^{\circ}\right)$, topographic curvature (-32 to +29$)$, digital elevation (350-2906 m a. s. 1.), internal relief (0-111 m for $3 \times 3$ pixels) and slope $\left(0^{\circ}\right.$ to $\left.61^{\circ}\right)$, short named $\mathbf{A}, \mathbf{C}, \mathbf{D}, \mathbf{I}$ and $\mathbf{S}$, respectively.

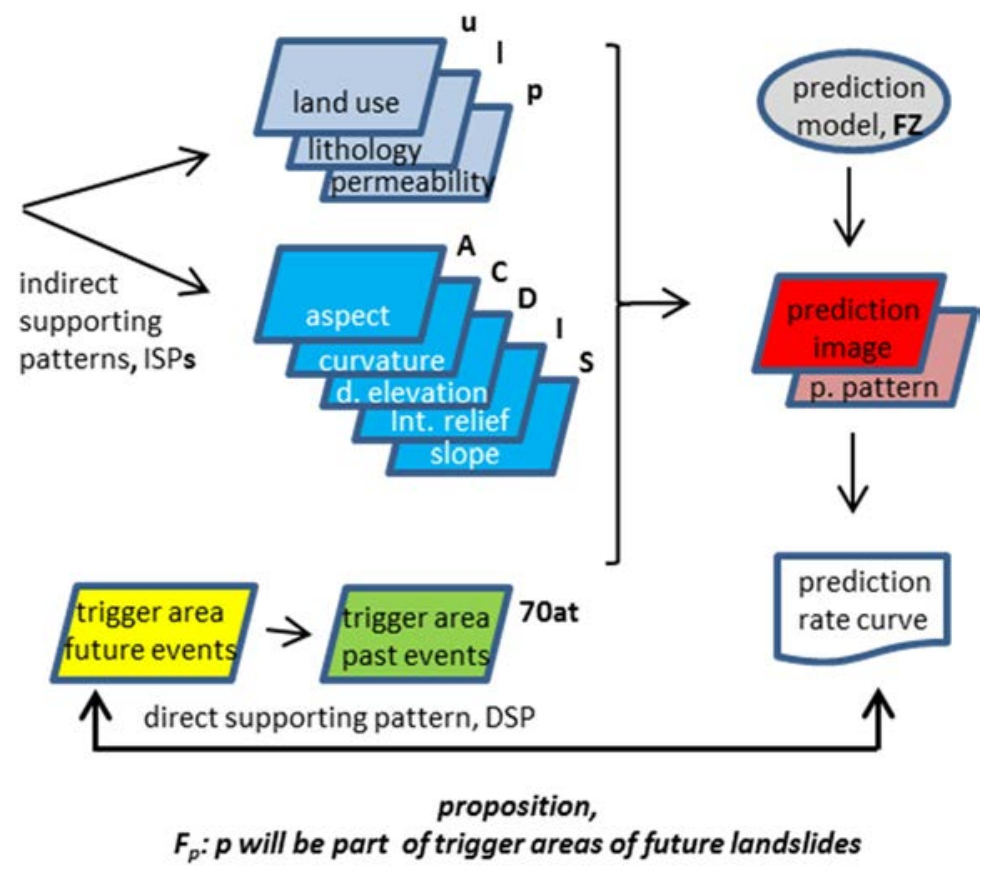

Figure 3: The spatial database of the Tirano South study area organized into direct supporting pattern, DSP (70at) and indirect supporting patterns ISPs (ulpACDIS) and, linking proposition, fuzzy set function, FZ, modelled prediction image, prediction pattern and prediction-rate curve. 
Table 1: Categorical ISPs: $\mathrm{u}_{1-23}$ land use classes, $1_{1-51}$, lithology units and $\mathrm{p}_{1-8}$ permeability classes. In bold the more supportive ones. (Source: Modified after [4].)

\begin{tabular}{|c|c|}
\hline $\begin{array}{c}\text { Land use, } \\
\mathrm{u}_{1-23}\end{array}$ & $\begin{array}{c}\text { Lithology, } \\
1_{19-51}\end{array}$ \\
\hline $\begin{array}{l}\text { 1, Rivers and channels; } \\
\text { 2, Rock and scree vegetation; } \\
\text { 3, Bedrock outcrops and surficial } \\
\text { deposits; } \\
\text { 4, Lakes, basins and surficial waters; } \\
\text { 5, Glaciers and perpetual snow; } \\
\text { 6, Bush and shrubs; } \\
\text { 7, Coniferous forest; } \\
\text { 8, Grassland and pasture; } \\
\text { 9, Broad-leaved forests; } \\
\text { 10, Urban areas and infrastructures; } \\
\text { 11, Mixed forests; } \\
\text { 12, Permanent meadows; } \\
\text { 13, Vegetated riverbeds; } \\
\text { 14, Beaches, sands and gravel areas; } \\
\text { 15, Degraded areas with different uses; } \\
\text { 16, Recent reforestations; } \\
\text { 17, Arable land with trees; } \\
\text { 18, Fruit tree and berry plantations; } \\
\text { 19, Vineyards; } \\
\text { 20, Arable land; } \\
\text { 21, Mineral extraction sites; } \\
\text { 22, Vegetated wet areas and peats; } \\
\text { 23, Waste disposition areas. }\end{array}$ & $\begin{array}{l}\text { 19, Vegetated deposit; } \\
\text { 20, Non vegetated deposit; } \\
\text { 21, Partially vegetated deposit; } \\
\text { 22, Dolomite; } \\
\text { 23, Eluvial deposits; } \\
\text { 24, Eluvial-colluvial deposits; } \\
\text { 25, Non-active partially vegetated scree slope; } \\
\text { 26, Active non-vegetated scree slope; } \\
\text { 27, Active partially vegetated scree slope; } \\
\text { 28, Non-active partially vegetated scree slope; } \\
\text { 29, Phyllites and clay-schists; } \\
\text { 30, Outcropping phyllites and clay-schists; } \\
\text { 31, Glaciers; } \\
\text { 32, Gneisses; } \\
\text { 33, Outcropping gneisses; } \\
\text { 34, Micaschists; } \\
\text { 35, Outcropping micaschists; } \\
\text { 36, Stadial moraine; } \\
\text { 37, Glacial deposit; } \\
\text { 38, Paleo-landslide; } \\
\text { 39, Quartzites; } \\
\text { 40, Outcropping quartzites; } \\
\text { 41, Effusive rocks; } \\
\text { 42, Outcropping effusive rocks; }\end{array}$ \\
\hline $\begin{array}{l}\qquad \begin{array}{l}\text { Lithology, } \\
\qquad 1_{1-18}\end{array} \\
\text { 1, Rock fall deposits; } \\
\text { 2, Debris flow deposits; } \\
\text { 3, Alluvial deposits; } \\
\text { 4, Wet areas; } \\
\text { 5, Unproductive areas; }\end{array}$ & $\begin{array}{l}\text { 43 Hypoabissal rocks; } \\
\text { 44, Outcropping hypoabissal rocks; } \\
\text { 45, Intrusive rocks; } \\
\text { 46, Outcropping intrusive rocks; } \\
\text { 47, Outcropping metamorphic carbonate rocks; } \\
\text { 48, Metamorphic carbonate rocks; } \\
\text { 49, Serpentinites; } \\
\text { 50, Outcropping serpentinites; } \\
\text { 51, Peat deposits. }\end{array}$ \\
\hline $\begin{array}{l}\text { 7, Claystones; } \\
\text { 8, Colluvial deposits; } \\
\text { 9, Conglomerates; }\end{array}$ & $\begin{array}{l}\text { Permeability, } \\
\mathrm{p}_{1-8}\end{array}$ \\
\hline $\begin{array}{l}\text { 10, Exposed conglomerate outcrops; } \\
\text { 11, Non-active vegetated talus; } \\
\text { 12, Active non-vegetated talus; } \\
\text { 13, Active partially vegetated talus; } \\
\text { 14, Non-active partly vegetated talus; } \\
\text { 15, Non-active vegetated talus; } \\
\text { 16, Active non vegetated talus; } \\
\text { 17, Moraine ridge; } \\
\text { 18, Fluvio-glacial deposit; }\end{array}$ & $\begin{array}{l}\text { 1, Cohesive units of high-medium permeability; } \\
\text { 2, Cohesive units with low permeability; } \\
\text { 3, Cohesive units with very low permeability; } \\
\text { 4, Non-cohesive units with high-medium } \\
\text { permeability; } \\
\text { 5, Non-cohesive units with low permeability; } \\
\text { 6, Non-cohesive units with very low permeability; } \\
\text { 7, Wetland - extremely low permeability; } \\
\text { 8, No data. }\end{array}$ \\
\hline
\end{tabular}


In Fig. 3 the DSP and the ISPs are used by the prediction model, based on a fuzzy set function, $\mathbf{F Z}$, to integrate their spatial relationships into a prediction image of high digital resolution with scores ranging between 0 and 1 , then transformed into a prediction pattern by ranking the scores into 200 classes in descending order, from 200 to 1. Fig. 4 shows how the predicted scores in the prediction image with values ranging between 0.00 and 0.28 were converted into 200 equal area ranks. The prediction scores are not considered interpretable beyond their equal area ranks.

Note in Fig. 3 the implied separation of the DSP into trigger areas of the past events and those of future events. As we will see later, such a separation allows assessing the predictive quality of the prediction pattern by the calculation of a prediction-rate curve by crossvalidation of prediction patterns [6]. In essence, it is the proposition that links DSP and ISPs into a mathematical expression to be supported as true or false by the evidence in the database.

\section{ASSUMPTIONS IN PREDICTION MODELLING}

\subsection{Concerning the database}

To support the proposition at the core of the prediction modelling, at least four assumptions need to be made. First one is that the database sufficiently represents the spatiotemporal characteristics of the hazardous process. Natural hazards, like the occurrence of landslides, are the results of gravity- and erosion-induced processes at the hearth surface, also influenced by tectonism and seismicity over large regions. Second assumption is that the ISPs do provide factors related with the landslides. It means that they represent at least in part the setting of the occurrence due to those processes or in other words the typical geomorphologic conditions in the study area. Third is that the DSP spatiotemporal distribution corresponds to a homogeneous dynamic type of occurrences that can be partitioned into younger and older sub-sets of events. And fourth assumption is that the spatiotemporal conditions of the older sub-set are similar or comparable to those of the younger sub-set thus enabling the prediction.

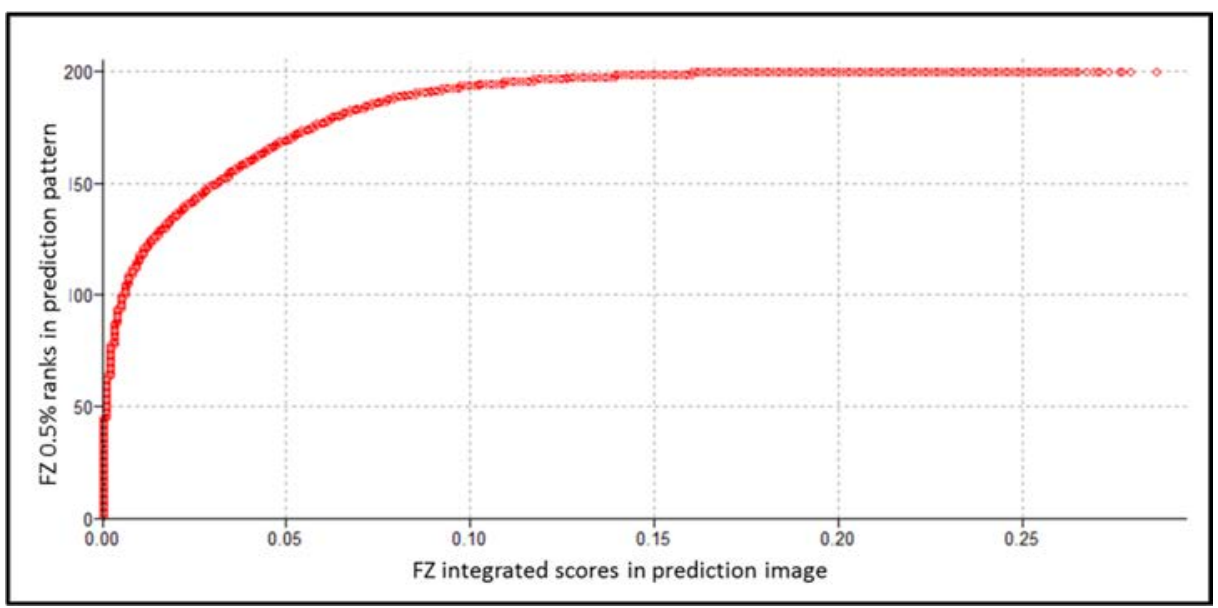

Figure 4: Relationships of sorted fuzzy set, FZ, prediction scores ranging from 0.00 to 0.28 and the corresponding $0.5 \%$ equal area ranks ranging from 1 to 200 . 
Obviously, it is implicit that, given a study area selected due to safety or risk assessment concern, it is up to expert's knowledge to set the area extension and confines, level of detail, cartographic scale and quality of DSP and ISP map data so that the four assumptions above are satisfied.

\subsection{Concerning spatial relationships}

Spatial prediction modelling, as a simplification of reality, sufficiently detailed and informative, must provide insight on the pattern-generating process via the spatial relationships extracted from the database. The most common way of exploring such relationships is to subdivide the study area into two parts: a smaller sub-area with the presence of the landslide occurrence (better if it is the presence of their trigger areas), and a much larger sub-area in which those occurrences are absent or unobserved. Statistics within the two sub-areas are then calculated in terms of respective distribution of ISP units characterizing the two separate conditions or settings. Even if it is technically impossible to have complete information on all occurrences and on all "non-occurrences" (there may still be a minority of occurrences in the sub-area considered to be free of occurrences) we have to assume that the statistics allow classifying the study area into classes of higher and lower likelihood of occurrence. It is the contrast established between the statistics from the two subareas that will be used in the modelling. A convenient measure of such a contrast is the empirical likelihood ratio, ELR. Figs 5, 6 and Table 2 provide examples of ELR values for categorical and continuous-field ISPs. Fig. 5(a) shows as an example, the histogram the normalized frequencies of the 51 lithology units, used as ISP, for the two sub-areas with presence and assumed absence of landslides. In Fig. 5(b) is the corresponding ELR value histogram. As a rule-of-thumb for the interpretation of ELR values, note that a value of 2 represents a frequency in the presence of landslides twice that in their absence within the study area. Fig. 6(a) shows, as another example, the density function of the slope angles in the presence and in the absence of the landslides and in Fig. 6(b) is the corresponding ELR function. Table 2 shows only the ELR values found most effective in establishing a contrast between presence and absence of landslides: two land use classes, eight lithology units and one permeability class with values $\geq 2$ (see description in bold in Table 1). Note also the supportive ranges of aspect, curvature ( 2 intervals), digital elevation ( 2 intervals), internal relief and slope.

Although it is not indispensable to have DSP and ISPs digitized with the same resolution, it is convenient because it simplifies both the computations and the interpretations. In the Tirano South database the landslide occurrence trigger areas were digitized to occupy 697 pixels of $20 \mathrm{~m}$ resolution. They cover the smaller sub-area used for modelling. The same resolution was used for the entire database of ISPs for $\boldsymbol{A}$ covering 646,091 pixels obviously co-registered with the pixels of the DSP. In it the larger sub-area of pixels to be classified covers $646,091-697=645394$ pixels, i.e., an area three orders of magnitude larger than that occupied by the 697 pixels.

\subsection{Concerning prediction models}

The mathematical model considers the ratios, ELR, like those shown in Table 2, for all individual pixels in the $\boldsymbol{A}$, transforms them into a score for each categorical unit and continuous-field value of the ISPs, and then combines them into an aggregated score classifying the $\boldsymbol{A}$ into levels of likelihood of occurrence. 

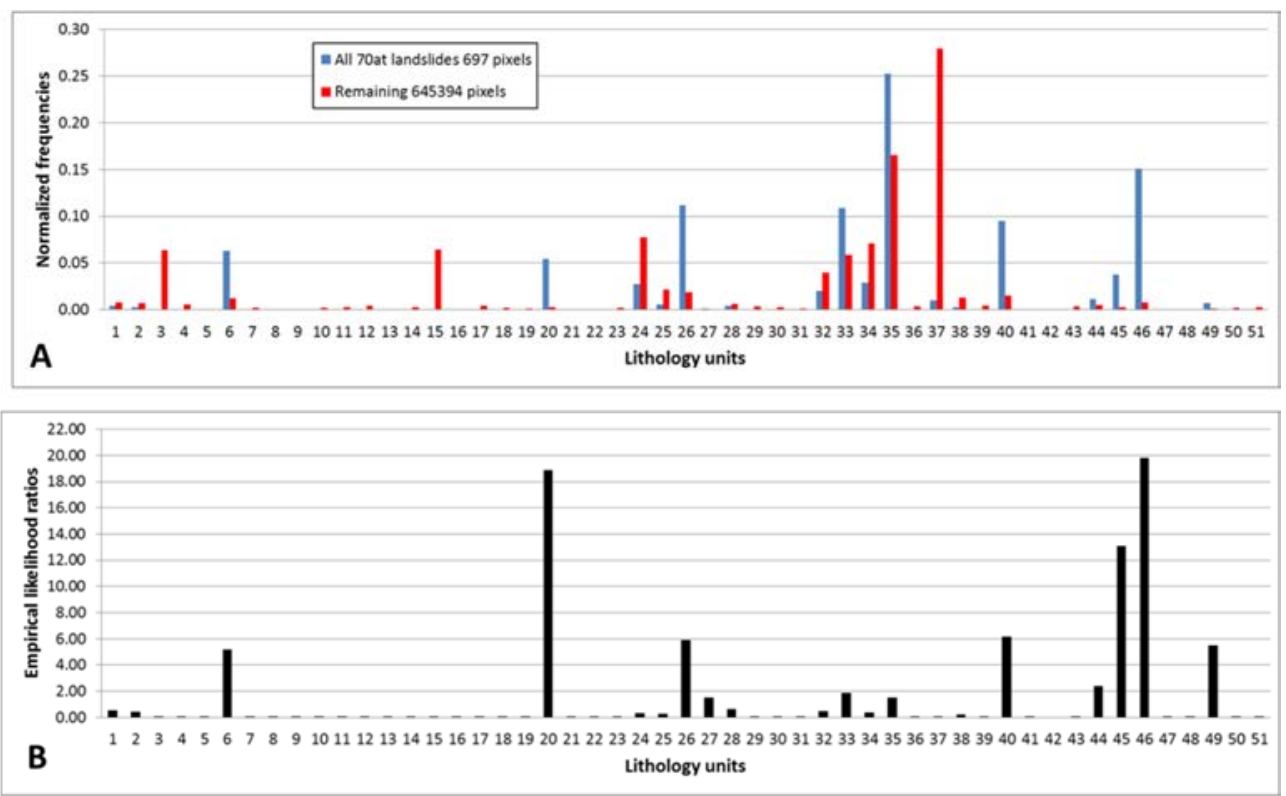

Figure 5: Histograms of normalized frequencies of lithology units in the presence and in the absence of landslides in the study area in (a) and corresponding empirical likelihood ratios in (b). See description of units in Table 1.
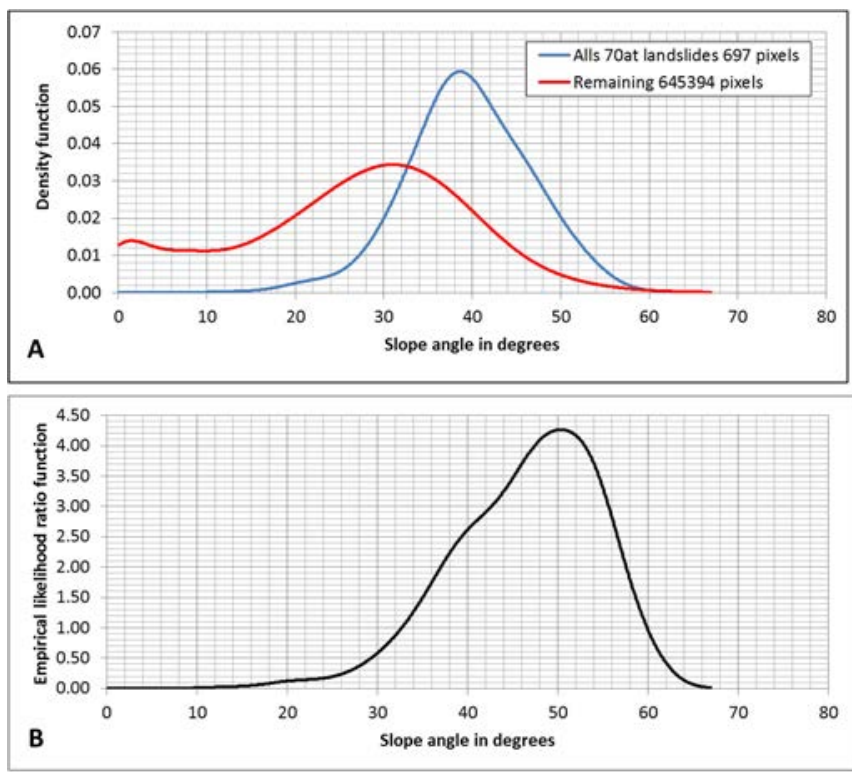

Figure 6: Density functions of slope angles in the presence and in the absence of landslides in the study area in (a) and corresponding empirical likelihood ratio function in (b). 
Table 2: Empirical likelihood ratio values for predictions using as DSP the distribution of the set of landslides, 70at with ulpACDIS as ISPs. Abbreviations for categorical ISPs are as in Table 1: $\mathrm{u}_{1-23}$ land use classes, $\mathrm{l}_{1-51}$ lithology units and $\mathrm{p}_{1-8}$ permeability classes. For the continuous field ISPs A, C, D, I and S, are aspect, curvature, digital elevation, internal relief and slope, respectively. Values are bold if the ELR $\geq 2.00$. In Italics is the corresponding range of classes, with maximum ratio in brackets. (Source: Modified after [4].)

\begin{tabular}{|l|l|}
\hline \multicolumn{1}{|c|}{ DSP and ISPs } & \multicolumn{1}{c|}{ Empirical likelihood ratio values } \\
\hline \multicolumn{1}{|c|}{ 70at } & \\
Land use & $\mathrm{u}_{2}=\mathbf{2 . 2 9}, \mathrm{u}_{3}=\mathbf{4 . 4 8} ;$ \\
Lithology & $1_{6}=\mathbf{5 . 1 9}, \mathrm{l}_{20}=\mathbf{1 8 . 9 1}, \mathrm{l}_{26}=\mathbf{5 . 8 9}, \mathrm{l}_{27}=1.51, \mathrm{l}_{33}=1.86,1_{35}=1.53$, \\
& $\mathrm{l}_{40}=\mathbf{6 . 1 5}, \mathrm{l}_{44}=\mathbf{2 . 4 1}, \mathrm{l}_{45}=\mathbf{1 3 . 1 0}, \mathrm{l}_{46}=\mathbf{1 9 . 7 9}, 1_{49}=\mathbf{5 . 5 2} ;$ \\
Permeability & $\mathrm{p}_{2}=1.92, \mathrm{p}_{3}=\mathbf{2 . 0 6}, \mathrm{p}_{4}=1.91$ \\
Aspect & $\geq 2: 168-198,(\max \mathbf{2 . 0 7}) ;$ \\
Curvature & $\geq 2:-24--7,(\max \mathbf{1 1 . 0 7}) ;+7-+17,(\max \mathbf{5 . 6 2}) ;$ \\
D. elevation & $\geq 2: 1737-2104,(\max \mathbf{4 . 2 4}) ; 2484-2629,(\max \mathbf{2 . 3 1}) ;$ \\
Int. relief & $\geq 2: 24-56,(\max \mathbf{5 . 9 7}) ;$ \\
Slope & $\geq 2: 37-57,(\max \mathbf{4 . 2 6})$ \\
\hline
\end{tabular}

A general assumption made in most spatial prediction models is that the ISPs are conditionally independent so that the scores obtained are not affected by redundancy. In the earth sciences, however, most ISPs are, to a large extent partial aspects of a spatial process so that they tend to be conditionally dependent. Such dependency is fundamental property of the spatial data and cannot be simply avoided or eliminated. Even if the dependence does not show numerically, or it is made not to show by numerical transformations, it still exists conceptually within the database because it is implicit in the data. Numerical elimination of such conditional dependence in the database may sacrifice the support from important ISPs [7]. For instance, we may want to compare the results of modelling with and without the presence of heavily conditionally-dependent ISPs and then decide on the level of alteration in the classification due to the dependence. The modelling here was applied using the software approach termed Spatial Target Mapping and discussed by Fabbri et al. [8]. It was programmed specifically for guiding in favourability modelling [2]-[5].

Let us now consider the Fuzzy Set function used for prediction modelling to generate the prediction patterns shown in Fig. 1. The study area $\boldsymbol{A}$ consists of $\mathrm{m}$ map data layers (h categorical data layers and k continuous-field data layers) with an occurrence data layer containing the distribution of the occurrences. In our case $\mathrm{h}=3$ and $\mathrm{k}=5$. Let us consider a pixel $p$ in $\boldsymbol{A}$. We have $\mathrm{m}$ evidences $\left(e_{j}(p), j=1, \cdots, m\right)$ at $p$, one for each of the $m$ data layers and the following proposition:

$$
\mathbf{T}_{\mathrm{p}} \text { : " } p \text { will be part of an occurrence." }
$$

We wish to define a favourability function (see Chung and Fabbri, 2001 [5]) at $p$ representing a degree of support for the proposition in (1) given the m evidences $\left(e_{j}(p), j=\right.$ $1, \cdots, m)$,

$$
\boldsymbol{f}\left(\mathrm{T}_{\mathrm{p}} \mid e_{j}(p), j=1, \cdots, m\right) \text { at each pixel } p \text { in } \boldsymbol{A} .
$$


Using the Fuzzy-Set's membership function (Zadeh, 1965; 1968; 1978) [9]-[11] we could write:

$$
\boldsymbol{f}\left(\mathrm{T}_{\mathrm{p}} \mid e_{1}(p), \cdots, e_{m}(p)\right)=\boldsymbol{\mu}\left\{\mathrm{T}_{\mathrm{p}} \mid e_{1}(p), \cdots, e_{m}(p)\right\}
$$

where $\boldsymbol{\mu}\left\{\mathrm{T}_{\mathrm{p}} \mid e_{1}(p), \cdots, e_{m}(p)\right\}$ is the membership function of the following corresponding fuzzy set:

$$
\mathbf{S}_{\mathrm{T} \mathrm{p}}=\left\{\left(p, \boldsymbol{\mu}\left\{\mathrm{T}_{\mathrm{p}} \mid e_{1}(p), \cdots, e_{m}(p)\right\}\right), p \in \boldsymbol{A}\right\}
$$

A fuzzy set $\mathbf{S}_{\mathrm{Tp}}$ consists of all the pixels where the proposition in (1) is "likely" to be true. The fuzzy set $\mathbf{S}_{\mathrm{Tp}}$ is uniquely defined by the membership function $\boldsymbol{\mu}\left\{\mathrm{T}_{\mathrm{p}} \mid e_{1}(p), \cdots, e_{m}(p)\right\}$. Once we have defined a membership function for each layer separately, from these $\mathrm{m}$ membership functions and the corresponding $\mathrm{m}$ fuzzy sets, we obtain the joint membership function and the corresponding fuzzy set, $\boldsymbol{\mu}\left\{\mathrm{T}_{\mathrm{p}} \mid e_{1}(p), \cdots, e_{m}(p)\right\}$ and $\mathbf{S}_{\mathrm{Tp}}$, discussed in eqns (3) and (4) using several fuzzy set operators. For the prediction pattern in Fig. 1 we have selected a gamma function operator with $\gamma=0.5$. With $\gamma=1$ we have an algebraic sum operator, with $\gamma=0$ we have an algebraic product operator and with $\gamma=0.5$ the combination of the two [5]).

Assumptions of conditional independence are implied at various steps in modelling, from transforming the original ELRs into scores of categorical and continuous ISPs to their separate aggregation and the aggregation of the two types of ISP. We have observed that in similar applications the presence of even a relatively high conditional dependence had only minor effect mostly on the lower ranks of a prediction pattern [12].

\section{INTERPRETATION AND EVALUATION OF PREDICTION PATTERNS}

The prediction pattern in Fig. 1 was obtained using the distribution of all the 70at occurrences as DSP. Therefore, it represents the most complete prediction. However, so far we do not know much about its predictive quality. To interpret the prediction capability, we would need to use a set of future occurrences and verify how well they are distributed across the higher ranks of the prediction pattern.

Since we do not have such a future set (the year of occurrence is not available in the database) we can pretend to obtain it by partitioning the 70at occurrences into a modelling or training sub-set and a cross-validation sub-set. In this case the latter would represent the "next" set of occurrences in time. There are many ways to do that, for instance spatially, randomly, sequentially or just arbitrarily. Suppose, that we sequentially exclude 7 occurrences out of the 70at, we can iterate prediction modelling and cross-validating 10 times to obtain 10 prediction patterns each one using 63 as DSP and verify by cross-validation the ranking of the remaining 7 occurrences. A prediction-rate curve is then obtained considering the prediction scores of all the 70at occurrences, as shown in Fig. 7 by the heavier black continuous line. To generate the diagram with the curve, 200 equal area ranks are constructed out of an ordered sequence of 646,091 prediction scores plotted on the horizontal axis. On the vertical axis we have the corresponding cumulative proportion of the 697 occurrence pixels for each rank of $0.5 \%$ of the study area. In the illustration we also have the curves for predictions that used as ISPs only ulp (in red), only ACDIS (in blue) with the one that used all ulpACDIS (in black). Obviously there is a difference of 3 orders of magnitude between the proportion values on the vertical axis and the ones on the horizontal one. For this reason the diagram of Fig. 7 is using a relatively shorter vertical axis. 


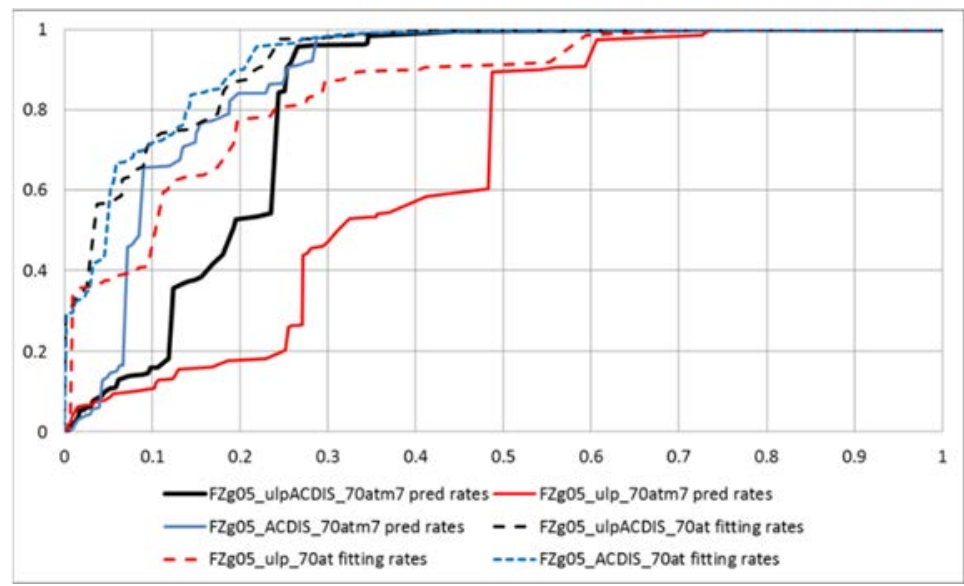

Figure 7: Cumulative curves of fitting-rates, broken lines, and prediction-rates, continuous lines, for different $\mathbf{F Z}$ prediction patterns using only categorical, red, or only continuous, blue, or categorical and continuous, black, ISPs. The prediction-rate curves are from 10 iterations of sequential exclusion of 7 occurrences. Explanation is in the text.

To interpret the black prediction-rate curve obtained by sequential exclusion of 7 occurrences, short named as FZg05_ulpACDIS_70atm7 (g05 for $\gamma=0.5$ and $\mathbf{m} 7$ for sequential exclusion of 7), observe that the top $10 \%$ ranks correspond to $16 \%$ of the occurrence pixels, the top $20 \%$ to the $53 \%$, the top $25 \%$ to the $87 \%$ and the top $30 \%$ to $98 \%$. Furthermore, note that the top $25 \%$ of the equal area ranks is the only significant part of the curve from a cost/benefit point of view. When the curve approximates the diagonal the occurrence pixels tend to be randomly distributed through the cumulative ranks that follow to the right.

In addition, the diagram shows as broken lines the corresponding fitting-rate curves in which all the non-partitioned 70at occurrences were used as DSP. They are all steeper than the prediction-rate curves. Clearly the blue prediction-rate curve from a prediction that used only the continuous field ISPs appears to be overfitting while the one that used only the categorical ISPs, is under fitting.

The black prediction-rate curve also shows a slow rise from the origin to the top $12 \%$ ranks followed by a first steep rise. It could indicate a non-homogeneous setting of some of the occurrences used as DSP. This is something worth exploring as we will see later on in further experiments. Generally, the fitting-rate curve provides information on the skewness of the classification and not on the predictive capability, which is displayed instead by the prediction-rate curve.

Besides generation of prediction-rate tables and curves the 10 prediction patterns can be subjected to statistical analyses to obtain what we have termed as a Target Pattern, in this case the aggregated expression of the median rank of the ten prediction ranks for each pixel. The Target Pattern is visually indistinguishable from the prediction pattern in Fig. 1, so that it is not shown here, however, from it we can compute the corresponding range of ranks. The range represents the degree of uncertainty of the assignment of each pixel to a given rank and is shown in Fig. 8(a) as ranking of ranges, termed Uncertainty Pattern, overlaid with the distribution of the 70at occurrences in black. Furthermore, we can threshold such a range 
image at a desired \% of lower range (variation) and use the set of pixels below the threshold to visualize only the corresponding pixels in the Target Pattern. This is shown in Fig. 8(b) as the Uncertainty/Target Combination Pattern for the 50\% lower uncertainty values. Considering the distribution of the 70at occurrences across the 50\% Combination Pattern, we can observe how a number of occurrences are located in areas with higher uncertainty of rank membership. In our example 47 of them correspond to lower uncertainty and 23 to higher uncertainty. Such distribution is an expression of degree of uncertainty of class membership and should contribute to the evaluation of the prediction pattern in Fig. 1. The wider is the range the higher is the uncertainty.
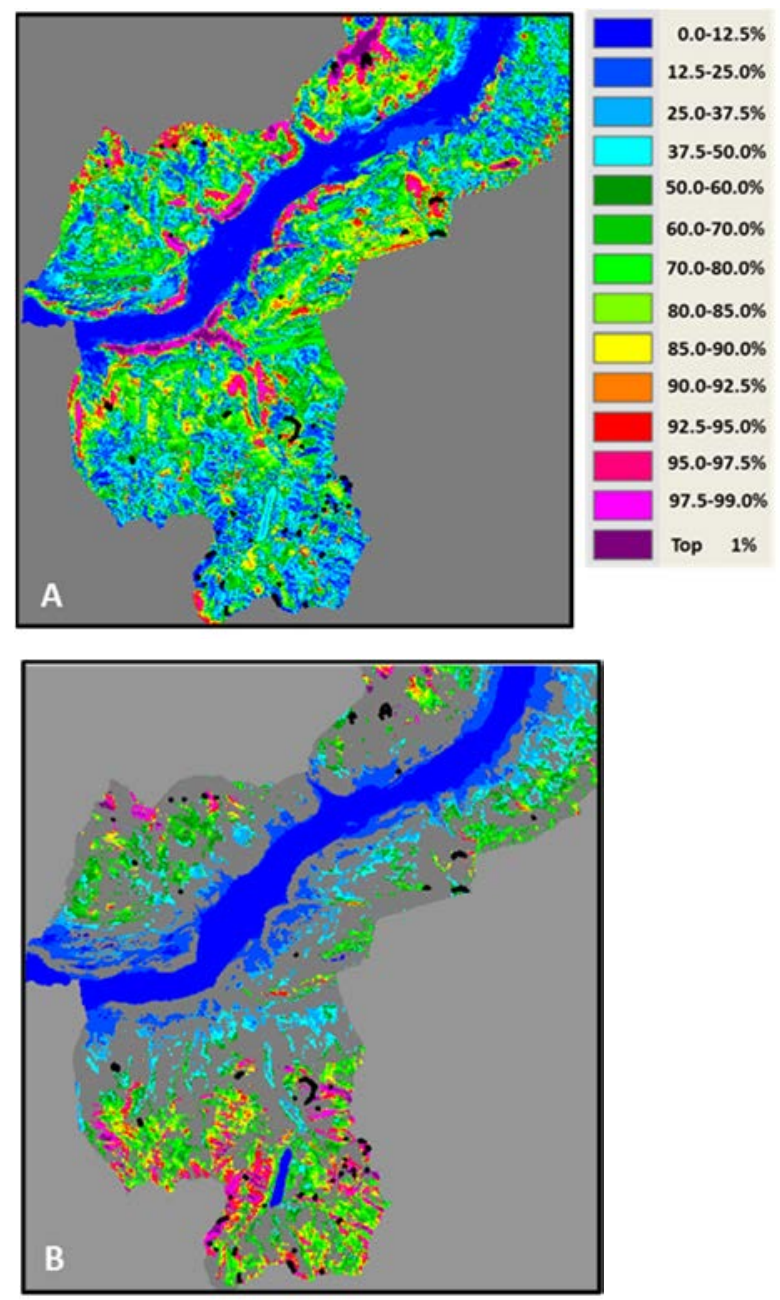

Figure 8: Fuzzy set function Uncertainty Pattern in (a) and 50\% Combination Pattern in (b), generated from the Target Pattern. It was obtained by a strategy of sequential exclusion of 7 occurrences from the 70at landslides, FZg05 ulpACDIS_70atm7. Occurrences in black and colours are the same as in Fig. 1; 23 out of $7 \overline{0}$ are in areas of higher uncertainty of rank membership. Explanation is in the text. 
Iterative processes of cross-validation can also be used to characterize the degree of similarity of setting of the occurrences in the study area represented in the database and possibly help in the interpretation of their support of the proposition in generating the prediction pattern. We have used another strategy, the one of sequential selection of one occurrence as DSP to obtain 69 prediction scores for the remaining cross-validated occurrences. A $70 \times 70$ array of prediction scores with null values in the diagonal is produced that was used to separate relatively well predicting/predicted occurrences, $\mathbf{W p p}$, from poorly predicting/predicted ones, Ppp. In our example we have arbitrarily selected as $\mathbf{W p p}$ those with prediction ranks within the top $10 \%$ when predicted by at least 20 occurrences while predicting at least 9 other occurrences within that top rank. This tentative selection produced 44atWpp, occupying 340 pixels, and 26atPpp, occupying 357 pixels.

The separation of the two groups enabled generating two distinct prediction patterns using either the 44atWpp or the 26atPpp as DSP. Even before obtaining the prediction patterns we considered the respective ELRs shown in Table 3. The support by the various categorical ISPs shows a clear separation of settings for lithology and permeability and land use for the 44atWpp patterns from those of the 26atPpp. Fig. 9 shows the prediction-rate curves produced by iterative processes of sequential selection of 4 from the 44atWpp and of 2 from the 26atPpp (11 and 13 iterations, respectively).

Table 3: Empirical likelihood ratio values for predictions using the two subsets of landslides, 44atWpp and 26atPpp with ulpACDIS as ISPs. Abbreviations for categorical ISPs are as in Table $2: \mathrm{u}_{1-23}$ the land use classes, $1_{1-51}$ the lithology units, $p_{1-8}$ the permeability classes. For the continuous field ISPs A, C, D, I and $\mathbf{S}$, are aspect, curvature, digital elevation, internal relief and slope, respectively. Values are bold if the ELR $\geq 2.00$. In Italics is the corresponding range of classes (with maximum ratio in brackets).

\begin{tabular}{|c|c|c|}
\hline $\begin{array}{c}\text { DSP } \\
\text { and ISPs }\end{array}$ & Empirical likelihood ratio values & \\
\hline $\begin{array}{l}\quad \text { 44atWpp } \\
\text { Land use } \\
\text { Lithology } \\
\text { Permeability } \\
\text { Aspect } \\
\text { Curvature } \\
\text { D. Elevation } \\
\text { Int. Relief } \\
\text { Slope }\end{array}$ & $\begin{array}{l}\mathrm{u}_{2}=\mathbf{2 . 9 7}, \mathrm{u}_{3}=\mathbf{8 . 9 4} ; \\
\mathrm{l}_{6}=\mathbf{1 0 . 4 0}, \mathrm{l}_{20}=\mathbf{5 . 0 1}, \mathrm{l}_{26}=\mathbf{1 2 . 0 9}, \mathrm{l}_{27}=\mathbf{3 . 0 9}, \mathrm{l}_{33}=1.31,1_{35}=0.64, \\
=0.38,1_{44}=0.00,1_{45}=\mathbf{2 6 . 8 7}, 1_{46}=\mathbf{4 0 . 5 9}, 1_{49}=0.00 ; \\
\mathrm{p}_{2}=0.71, \mathrm{p}_{3}=\mathbf{3 . 2 5}, \mathrm{p}_{4}=\mathbf{2 . 5 6} \\
\geq 2: 141-213,(\max \mathbf{3 . 7 7}) ; \\
\geq 2:-5.66-+3.25,(\max \mathbf{5 . 3 3}) ;+18.00-+28.00,(\max \mathbf{6 . 8 6}) ; \\
\geq 2: 1775-2149,(\max \mathbf{5 . 1 2}) ; 2399-2736,(\max \mathbf{4 . 0 6}) ; \\
\geq 2: 23.38-58.24,(\max \mathbf{1 1 . 7 5}) ; \\
\geq 2: 36.45-55.00,(\max \mathbf{3 . 9 3})\end{array}$ & 140 \\
\hline $\begin{array}{l}\text { 26atPpp } \\
\text { Land use } \\
\text { Lithology } \\
\text { Permeability } \\
\text { Aspect } \\
\text { Curvature } \\
\text { D. elevation } \\
\text { Int. relief } \\
\text { Slope }\end{array}$ & $\begin{array}{l}\mathrm{u}_{2}=1.64, \mathrm{u}_{3}=0.23 ; \\
\mathrm{l}_{6}=0.23, \mathrm{l}_{20}=\mathbf{3 1 . 9 9}, \mathrm{l}_{26}=0.00, \mathrm{l}_{27}=0.00,1_{33}=\mathbf{2 . 3 9}, 1_{35}=\mathbf{2 . 3 8} \\
=\mathbf{1 1 . 6 4}, 1_{44}=\mathbf{4 . 7 1}, 1_{45}=0.00,1_{46}=0.00,1_{49}=\mathbf{1 0 . 7 8} \\
\mathrm{p}_{2}=\mathbf{3 . 0 6}, \mathrm{p}_{3}=0.92, \mathrm{p}_{4}=1.30 \\
\geq 2: 0-17,(\max \mathbf{2 . 2 5}) ; 221-270,(\max \mathbf{3 . 2 9}) \\
\geq 2:-17.35--3.42,(\max \mathbf{3 1 . 9 9}) ;+18.59-+27.00,(\max \mathbf{4 . 9 5}) \\
\geq 2: 1676-2048,(\max \mathbf{3 . 6 9}) ; \\
\quad \text { at } 17.94(\max 1.77) \\
\geq 2: 37.85-59.43,(\max \mathbf{5 . 0 2})\end{array}$ & 140 \\
\hline
\end{tabular}




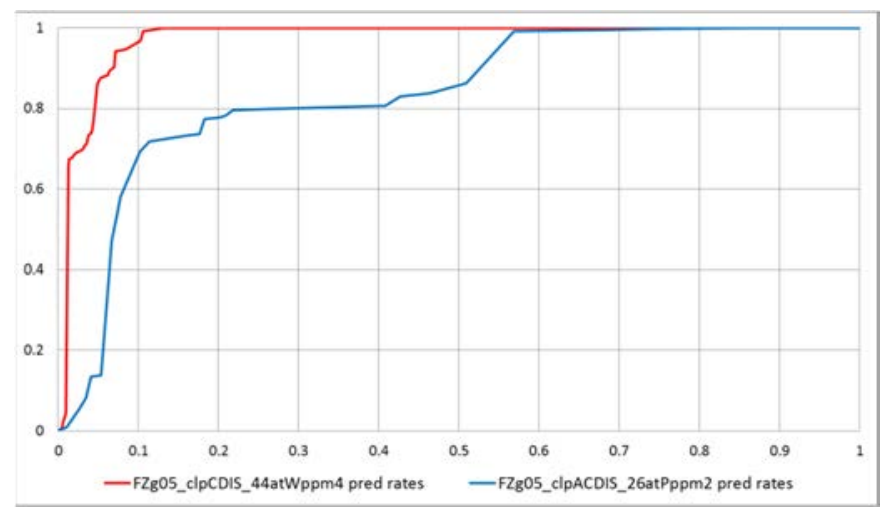

Figure 9: Prediction-rate curves from strategies FZg05_ulpACDIS_44atWppm4 in red and FZg05_ulpACDIS_26atPppm2 in blue.

Clearly the increase in homogeneity or similarity between the occurrences in each sub-set has changed the appearance of the prediction-rate curves! The curve from iterative crossvalidation strategy FZg05_ulpACDIS_44atWppm4, in red, shows that the top 5\% of the study area classified as hazardous contain $86 \%$ of the 340 pixels while the top $10 \%$ contain 96\%. The blue curve, from strategy FZg05_ulpACDIS_26atPppm2 shows that the top 5\% contains $14 \%$ while the $10 \%$ contains $69 \%$ and the top $20 \%$ contains $78 \%$.

But, what will the appearance be of the corresponding prediction patterns or Target Patterns? Fig. 10(a) shows the Target Pattern for the 44atWpp while Fig. 10(b) that for the 26atPpp. Here too the respective occurrence distributions are overlaid on the patterns. The visual difference in the patterns indicates the possible existence of at least two different settings: land use classes, lithology units and digital elevations contribute to the separation. They may be due to the existence of significantly different dynamic types of occurrences or just be part of the variability of a broad setting. The interpretation of the patterns becomes now the task of a specialist geomorphologist to relate them to the process generating the mass movements in the study area and eventually introduce the likelihood of occurrence in risk assessment.

\section{CREDIBILITY OF PREDICTION PATTERNS AND CONCLUDING REMARKS}

We can now reconsider the initial prediction pattern shown in Fig. 1 under a more informed new light. We have generated a prediction pattern modelling with a fuzzy set function the landslide susceptibility. For this in the Tirano South study area we have used the distribution of 70 active landslide occurrences and 8 categorical and continuous field digital maps. The prediction pattern has been disassembled into visual components, relative rankings and assumptions on data, spatial relationships, and modelling strategies. It was in order to gain confidence on the credibility of the database for prediction purpose. Measures of prediction capability and uncertainty of rank membership assured pattern interpretability. The homogeneity or congruity of the setting of the active landslides was tested by a strategy of sequential selection of one occurrence to generate 70 prediction patterns, one for each occurrence used for modelling, each providing 69 prediction rates for the remaining occurrences. In the study area a possible second type of landslide is postulated, should it be of value in practical decision-making for safer land use planning. We have gained confidence in the properties of the database and in its prediction patterns after many experiments. We 
have indicated the assumptions about the time/space distribution of the known hazardous events, the usefulness and completeness of the spatial database, the spatial relationships and the model for establishing and integrating them including aspects of representation of the likelihood levels as equal area ranks.

Relevant questions now are the following. Is our database worth the analysis and useable for land use planning and risk analysis? Do we know enough now about the database to apply some fine-tuning working on a variety of options in the analytical steps? What are the relationships between process knowledge and prediction pattern? How to separate the different degrees of certainty in our prediction patterns? What is at stake with spatial prediction modelling should we be reluctant to represent uncertainty levels?
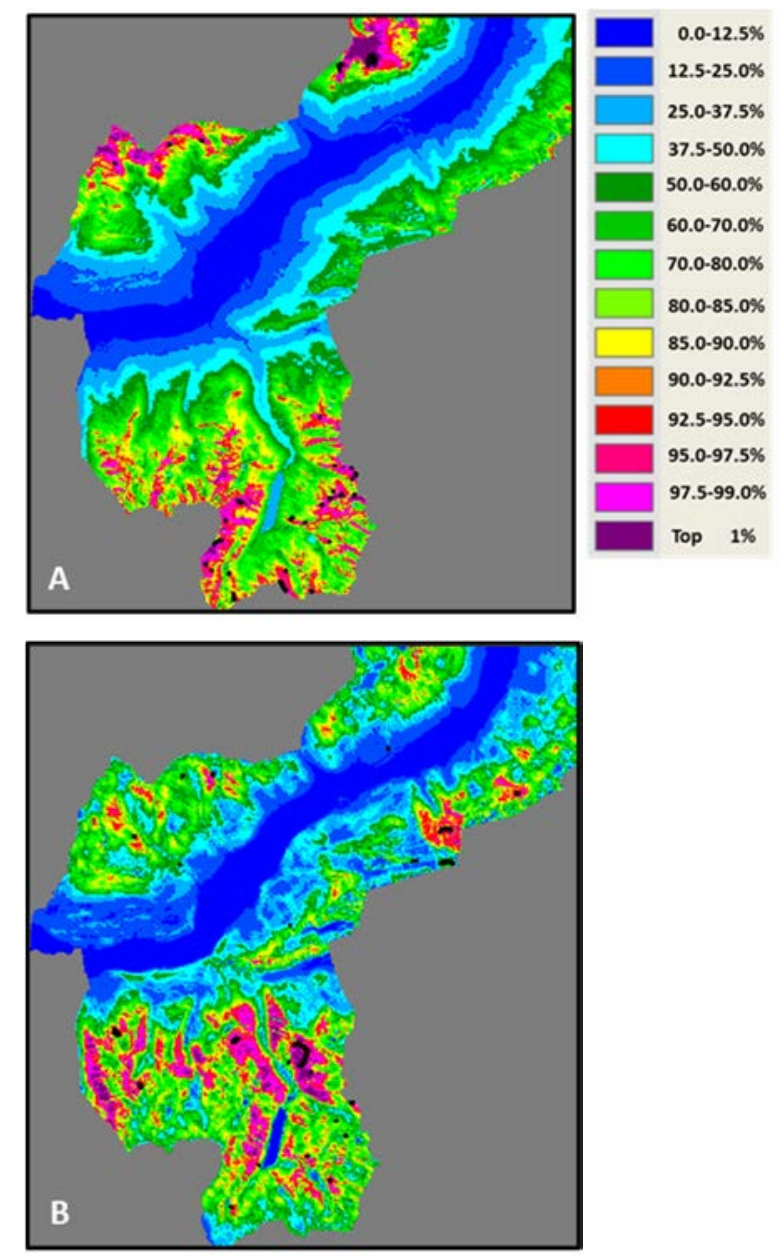

Figure 10: Target Patterns for two subsets of landslide occurrences obtained by a strategy of sequential selection of 1 out of 70at occurrences. (a) The pattern from 44atWpp as DSP using FZg05_ulpACDIS_44atWppm4 as iterative crossvalidation strategy; (b) That from 26atPpp as DSP using FZg05_ulpACDIS_26at Pppm2 as strategy. Occurrences in black and colours are the same as in Fig. 1. Explanation is in the text. 
We are now in the position to attempt providing answers to those questions at least within the realm of the study area. The answers do not necessarily require new unexplored study areas or newer fancier models but certainly they do need to get into deeper interpretations of study areas and modelling results. Should a prediction pattern appear unsatisfactory from a physical point of view, expert's knowledge can be used to tentatively modify DSP and ISP classes, units and values with updates and corrections for repeating the analyses and verifying their effects [5]. The dissection of a prediction pattern (hazard map?) leads to the confirmation of its credibility based on the confidence gained by the comprehension of all elements integrated and assumptions made in its construction. Such a dissection should be considered of general importance in any modelling procedure. Let us not forget that in modelling resource exploration, another common application area of favourability modelling, we encounter uncertainties that are probably much higher than those in modelling natural hazards! Many of the aspects discussed in this contribution are frequently ignored or hidden in hazard and risk studies.

\section{REFERENCES}

[1] Sangalli, L., Predisposizione dei versanti ai movimenti di massa attraverso curve di tasso di predizione. Studio statistico dell'area di Tirano Sud (Predisposition of slopes to mass movements through prediction-rate curves. Statistical study of the Tirano South area). MS thesis, DISAT, Univ. of Milano-Bicocca: Milan, Italy, 91, 2008.

[2] Chung, C.-J. \& Fabbri, A.G., Representation of geoscience data for information integration. Formerly Non-renewable Resources, Now Natural Resources Research, 2(2), pp. 122-139, 1993.

[3] Chung, C.-J., Using likelihood ratio functions for modelling the conditional probability of occurrence of future landslides for risk assessment. Computers \& Geosciences, 32(8), pp. 1052-1065, 2006.

[4] Fabbri, A.G., Cavallin, A., Patera, A., Sangalli, L. \& Chung, C.-J., Comparing patterns of spatial relationships for susceptibility prediction of landslide occurrences. Advancing Culture of Living with Landslides. Vol. 2, Advances in Landslide Science, Set 2., eds M. Mikoš, B. Tiwari, Y. Yin \& K. Sassa, Springer International Publishing AG: Cham, Switzerland, pp. 1135-1144, 2017.

[5] Chung, C.-J. \& Fabbri, A.G., Prediction models for landslide hazard using fuzzy set approach. Geomorphology and Environmental Impact Assessment, eds M. Marchetti \& V. Rivas, Balkema: Rotterdam, pp. 31-47, 2001.

[6] Chung, C.F. \& Fabbri, A.G., Validation of spatial prediction models for landslide hazard mapping. Natural Hazards, 30(3), pp. 451-472, 2003.

[7] Fabbri, A.G., Poli, S., Sterlacchini, S., Cavallin, A. \& Chung, C.-J., Uncertainty of class membership in spatial prediction modeling: follow-up study to an application to complex landslides. Proceedings of IAMG 2011, Mathematical Geosciences at the Crossroads of Theory and Practice, Salzburg, Austria, 5-9 Sept. 2011, p. 10, 2011.

[8] Fabbri, A.G., Patera, A. \& Chung, C.-J., Spatial target mapping: an approach to susceptibility prediction based on iterative cross-validations. Proceedings of IX Simposio Nacional sobre Taludes y Laderas Instables, Santander Spain 27-30 Jun. 2017, eds E. Alonso, J. Corominas \& H. Hürlimann, Centre Internacional de Mètodes Numèrics en Enginyeria (CIMNE): Barcelona, pp. 468-479, 2017.

[9] Zadeh, L.A., Fuzzy sets. IEEE Information and Control, 8, pp. 338-353, 1965.

[10] Zadeh, L.A., Probability measures of Fuzzy events. Journal of Mathematical Analysis and Applications, 10, pp. 421-427, 1968. 
[11] Zadeh, L.A., Fuzzy sets as a basis for a theory of possibility. Fuzzy Sets and Systems, 1(3), pp. 3-28, 1978.

[12] Fabbri, A.G., Poli, S., Patera, A., Cavallin, A. \& Chung, C.-J., Estimation of information loss when masking conditional dependence and categorizing continuous data, further experiments on a database for spatial prediction modelling in Northern Italy. Proceedings of 15th Annual Conference of the International Association for Mathematical Geosciences, Madrid, 2-6 Sep., p. 4, 2013. 